\title{
On sets of $n$ points in general position that determine lines that can be pierced by $n$ points
}

\author{
Chaya Keller* $\quad$ Rom Pinchasi ${ }^{\dagger}$
}

\begin{abstract}
Let $P$ be a set of $n$ points in general position in the plane. Let $R$ be a set of $n$ points disjoint from $P$ such that for every $x, y \in P$ the line through $x$ and $y$ contains a point in $R$ outside of the segment delimited by $x$ and $y$. We show that $P \cup R$ must be contained in a cubic curve.
\end{abstract}

\section{Introduction}

A beautiful result of Motzkin [Mot67, Rabin, and Chakerian [Cha70] states that any set of non-collinear red and blue points in the plane determines a monochromatic line. Grünbaum and Motzkin Grü75] initiated the study of biased coloring, that is, coloring of the points such that no purely blue line is determined. The intuition behind this study is that if the number of blue points is much larger than the number of red points, then unless the set of blue points is collinear, the set of blue and red points should determine a monochromatic blue line.

The same problem was independently considered by Erdős and Purdy [EP78] who stated it in a slightly different way.

Let $P$ be a set of $n$ points in the plane. Erdös and Purdy asked the following question in [EP78]: Assume a set $R$ of points in the plane is disjoint from $P$ and has the property that every line determined by $P$ passes through a point in $R$. How small can be the cardinality of $R$ in terms of $n$ ?

Clearly, if $P$ is contained in a line, then $R$ may consist of just one point. Therefore, the question of Erdös and Purdy is about sets $P$ that are not collinear. The best known lower bound for this question is given in [Pin13, where it is shown that $|R| \geq n / 3$.

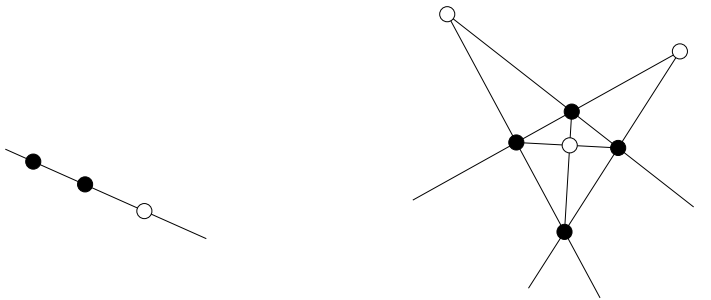

Figure 1: Constructions with $|R|=n-1$ for $n=2,4$. The points in $P$ are colored black while the points in $R$ are colored white.

In EP78 Erdös and Purdy considered the same problem in the case where the set $P$ is in general position in the sense that no three points of $P$ are collinear. In this case if $n$ is odd the

\footnotetext{
*Mathematics Department, Technion - Israel Institute of Technology, Haifa 32000, Israel. chayak@technion.ac.il. Research partially supported by Grant 409/16 from the Israel Science Foundation.

${ }^{\dagger}$ Mathematics Department, Technion - Israel Institute of Technology, Haifa 32000, Israel. room@tx.technion.ac.il. Research partially supported by Grant 409/16 from the Israel Science Foundation.
} 
tight bound $|R| \geq n$ is almost trivial because every point in $R$ may be incident to at most $\frac{n-1}{2}$ of the $\left(\begin{array}{l}n \\ 2\end{array}\right)$ lines determined by $P$. To observe that this bound is tight let $P$ be the set of vertices of a regular $n$-gon and let $R$ be the set of $n$ points on the line at infinity that correspond to the directions of the edges (and diagonals) of $P$. This construction is valid also when $n$ is even.

If $n$ is even, a trivial counting argument shows that $|R|$ must be at least $n-1$. This is because every point in $R$ may be incident to at most $n / 2$ lines determined by $P$. This trivial lower bound for $|R|$ is in fact sharp in the cases $n=2$ and $n=4$, as can be seen in Figure 1 . Is the bound $|R| \geq n-1$ sharp also for larger values of $n$ ?

The following theorem proves a conjecture attributed to Erdős and Purdy [EP78]. This conjecture was addressed in many papers (see, e.g., [BMP05, Chapter 7.3] and the references therein).

Theorem 1.1 ([ABK ${ }^{+} 08$, Mil18, $\left.\left.\mathrm{Pin} 18, \mathrm{PP} 19\right]\right)$. Let $P$ is a set of $n$ points in general position in the plane and, where $n>4$ is even. Assume $R$ is another set of points disjoint from $P$ such that every line through two points of $P$ contains a point from $R$. Then $|R| \geq n$.

Theorem 1.1 was first proved in $\left.\mathrm{ABK}^{+} 08\right]$ (see Theorem 8 there), as a special case of the solution of the Magic Configurations conjecture of Murty [Mur71. The proof in $\mathrm{ABK}^{+} 08$ ] contains a topological argument based on Euler's formula for planar maps and the discharging method. An elementary (and long) proof of Theorem 1.1 was given by Milićević in [Mil18. An algebraic proof of Theorem 1.1] is given in [Pin18]. Probably the "book proof" of the Theorem 1.1 can be found in PP19.

Theorem 1.1 was proved also over $\mathbb{F}_{p}$ by Blokhuis, Marino, and Mazzocca [BMM14].

As we have seen, there are constructions of sets $P$ of $n$ points in general position and sets $R$ of $n$ points not in $P$, such that every line determined by $P$ passes through a point in $R$. One major question that arises here is to characterize those sets $P$ in general position for which there exists a set $R$ with $|R|=|P|$ such that every line that is determined by $P$ passes through a point in $R$.

Already in [EP78] Erdös and Purdy drew a connection between the problem of finding a small set $R$ that pierces all the lines determined by a set $P$ and another well-known problem, raised by Scott [Sco70] and called 'the slopes problem':

What is the minimal number of directions spanned by a set $P$ of $n$ non-collinear points in the plane?

This is equivalent (by a projective transformation) to finding a set $R$ of minimum size that is contained in a line, and pierces all lines determined by a set $P$ of $n$ non-collinear points.

The slopes problem was solved by Ungar [Ung82, in a beautiful proof. Ungar showed that the minimum number of distinct direction of lines determined by a set of $n$ non-collinear points in the plane is $2\lfloor n / 2\rfloor$, and the bound is tight, e.g., for the vertex set of a regular $n$-gon for $n$ even, and for the vertex set of a regular $(n-1)$-gon plus its center point, for $n$ odd.

The extremal configurations for the slopes problem, i.e., sets of $n$ non-collinear points that span exactly $n-1$ directions, were studied in a series of papers of Jamison and Hill (see Jam85. and the references therein). They found four infinite families of configurations and 102 sporadic examples, and their list is conjectured to be complete for $n \geq 49$.

Elekes [Ele99] studied 'almost extremal' configurations, for which at most $c n$ directions are spanned. He provided a characterization of all such configurations with at least $c^{\prime} n$ points lying on a line and at least $c^{\prime \prime} n$ points lying outside that line. In the same paper, Elekes conjectured that for any $m \geq 6$ and $c>0$, there exists $n_{0}$ such that any set of $n \geq n_{0}$ non-collinear points in the plane that determines at most $c n$ directions, contains at least $m$ points that lie on a quadratic curve. This conjecture is still wide open, even for $m=6$.

When $P$ is assumed to be in general position in the sense that no three of its points are collinear, then the slopes problem becomes almost trivial. Every set of $n$ points in general position in the plane determines at least $n$ distinct directions for $n \geq 3$. 
Extremal and almost-extremal configurations for the slopes problem were studied also in the case where the point set in question is in general position. Jamison [Jam86 provided a complete characterization of the extremal configurations in this case, showing that if $P$ is a set of $n$ points in general position in the plane that determines exactly $n$ distinct directions of spanned lines then $P$ is an affine image of vertex set of a regular $n$-gon. In particular $P$ is contained in a quadric and because in this case the set $R$ is collinear, then $P \cup R$ is contained in a cubic curve in the plane. This simple observation will directly be related to the main result in this paper.

Jamison conjectured that if a set $P$ of $n$ points in general position in the plane determines precisely $2 n-c$ distinct direction where $c_{0} \leq c \leq n$ for an absolute constant $c_{0}$, then $P$ is obtained, up to an affine transformation, from a regular $(2 n-c)$-gon by omitting $n-c$ of its vertices. Recently, Pilatte Pil18] confirmed this conjecture for the case where $P$ determines precisely $n+1$ distinct directions.

We conjecture the following

Conjecture 1.2. Let $P$ be a set of $n$ points in general position and and let $R$ be a set of $n$ points disjoint from $P$. If every line determined by $P$ passes through a point in $R$, then $P \cup R$ is contained in a cubic curve.

It would be tempting to conjecture an even much more far reaching connection between the structure of $P$ and the cardinality of $R$, namely that if $|R|=O(|P|)$, then "many" points of $P$ lie on some low degree (cubic?) algebraic curve. This would correspond to the conjecture of Elekes mentioned above.

The main result in this paper is a proof of Conjecture 1.2 under the additional assumption that the line through every two points $x, y \in P$ contains a point from $R$ that lies outside of the segment determined by $x$ and $y$.

Theorem 1.3. Suppose $P$ is a set of $n$ points in general position in the plane and $R$ is another set of $n$ points, disjoint from $P$. If for every $x, y \in P$ there is a point $r \in R$ on the line through $x$ and $y$ and outside the interval determined by $x$ and $y$, then $P \cup R$ is contained in a cubic curve.

Theorem 1.3 generalizes in particular the characterization of Jamison in Jam86 for sets in general position that determine precisely $n$ distinct directions. Indeed, suppose a set $P$ of $n \geq 4$ points in general position in the plane determines precisely $n$ distinct directions, then there exists a collinear set $R$ of $n$ points (on the line at infinity) such that every line determined by two points $x$ and $y$ in $P$ contains a point of $R$. This point of $R$ must lie outside the segment delimited by $x$ and $y$ because $R$ is contained in the line at infinity. By Theorem $1.3 P \cup R$ is contained in a cubic $C$. Because $n \geq 4, C$ contains at least 4 collinear points on the line at infinity and therefore contains all the line at infinity. It follows now that $C$ is a union of a quadric and the line at infinity. Therefore, $P$ must be contained in a quadric.

The proof of Theorem 1.3 has two steps. In the first step, that is more of a combinatorial nature, we use our assumption that the point of $R$ collinear with two points $x$ and $y$ in $P$, lies outside of the segment delimited by $x$ and $y$. We show that in such a case the points of $P$ must lie in convex position. Moreover, the structure of collinearities in $P \cup R$ is uniquely determined: if we denote the points of $P$ by $x_{0}, \ldots, x_{n-1}$ in the cyclic order in which they are arranged in convex position, then one can rename the points in $R$ by $r_{0}, \ldots, r_{n-1}$ such that two points $x_{i}$ and $x_{j}$ in $P$ are collinear with $r_{k}$ iff $i+j+k=0$ modulo $n$. In the second step of the proof we use this information and follow the footsteps of Green and Tao in GT13, where the main algebraic ingredient is Chasles' theorem (see below), to conclude that $P \cup R$ is contained in some cubic curve. 


\section{Proof of Theorem 1.3}

Let $P^{\prime}$ denote the set of points in $P$ that are extreme on the convex hull of $P$. Let $k=\left|P^{\prime}\right|$. We first show that there are precisely $k$ points of $R$ outside of the convex hull of $P$. Fix a point $x$ in $P$. For every $y \in P \backslash\{x\}$ there is a point in $r \in R$ collinear with $x$ and $y$ that lies outside of the segment delimited by $x$ and $y$. In principle there can be more than one such point $r$. We choose one and say that it is relevant for $x$. Observe that for every $x$ in $P$ there is precisely one point in $R$ that is not relevant for $x$.

As a consequence, a line $\ell$ through two points in $P$ may contain only one or two points of $R$. This is because if it contains three, then for each of the two points of $P$ on $\ell$ there are at least two points of $R$ that are not relevant for it, contrary to our observation.

For every two points $x, y \in P^{\prime}$ it is true that the point in $R$ collinear with $x$ and $y$ must lie outside of the convex hull of $P$. Therefore, by Theorem 1.1 the number of points of $R$ outside the convex hull of $P$ must be at least $k$. On the other hand, let $R^{\prime}$ denote the set of all points of $R$ outside the convex hull of $P$. For every point $r \in R^{\prime}$ there are two lines through $r$ that are tangent to the convex hull of $P$. Among all those lines, let $a$ denote the number of the lines that support the convex hull of $P$ at a single vertex. Such a line must pass through precisely one point of $R^{\prime}$. This is because any point of $R^{\prime}$ on this line is not relevant for the unique point of $P^{\prime}$ on this line and for every point of $P$ there is precisely one point of $R$ that is not relevant for it. Notice that $a \leq 2\left|R^{\prime}\right|-k$ because there are $k$ lines supporting the convex hull of $P$ at an edge and each such line must contain at least one point of $R^{\prime}$. Let $b$ denote the number of lines that support the convex hull of $P$ at an edge and contain only one point of $R$. Let $c$ denote the number of lines that support the convex hull of $P$ at an edge and contain precisely two points of $R$. We have $a+b+2 c=2\left|R^{\prime}\right|$ and $b+c=k$.

It follows that the number of times a point in $R^{\prime}$ is not relevant for a point in $P^{\prime}$ is equal to $a+2 c$. This should be at most the cardinality of $P^{\prime}$, namely $k$.

Therefore, $k \geq a+2 c=2\left|R^{\prime}\right|-b \geq 2\left|R^{\prime}\right|-b-c=2\left|R^{\prime}\right|-k$ This readily implies $\left|R^{\prime}\right| \leq k$.

By showing that $\left|R^{\prime}\right| \geq k$ and $\left|R^{\prime}\right| \leq k$, we conclude that $\left|R^{\prime}\right|=k$. Therefore, $c$ must be equal to 0 . Moreover, for every point $p^{\prime} \in P^{\prime}$ there is a point $r^{\prime} \in R^{\prime}$ that is not relevant for it and the line through $p^{\prime}$ and $r^{\prime}$ is tangent to the convex hull of $P^{\prime}$ and supports it at the point $p^{\prime}$ alone.

We will now show that $P^{\prime}=P$. Assume not and let $P^{\prime \prime}$ be the set of extreme vertices of $P \backslash P^{\prime}$. We claim that no point of $R$ can lie inside the convex hull of $P^{\prime}$ and outside the convex hull of $P^{\prime \prime}$. Indeed (see Figure 2 for an illustration), if $r \in R$ is such a point, consider a line $\ell$ separating $r$ and $P^{\prime \prime}$. It must be that there is a point $p^{\prime} \in P^{\prime}$ in the half-plane bounded by $\ell$ and not containing $P^{\prime \prime}$, for otherwise $r$ cannot belong to the convex hull of $P^{\prime}$. Notice that $r$ cannot be relevant for $p^{\prime}$. This is a contradiction because there is already a point in $R^{\prime}$ that is not relevant for $p^{\prime}$.

Now take any point $p^{\prime} \in P^{\prime}$ and consider a line $\ell$ through $p^{\prime}$ that supports the convex hull of $P^{\prime \prime}$ at a point $p^{\prime \prime} \in P^{\prime \prime}$. The line $\ell$ must contain a point in $r \in R$. The point $r$ cannot lie inside the convex hull of $P^{\prime}$ because there is no point of $R$ inside the convex hull of $P^{\prime}$ and outside the convex hull of $P^{\prime \prime}$. Therefore, $r$ must be in $R^{\prime}$. But then the line $\ell$ through $r$ and $p^{\prime}$ contains only one point of $P^{\prime}$ while it is not a tangent line of the convex hull of $P^{\prime}$.

We conclude that $k=n, P=P^{\prime}$, and $R=R^{\prime}$. That is, the points of $P$ are in convex position and the points of $R$ lie outside the convex hull of $P$. We will now show that the collinear triples of points two from $P$ and one from $R$ are uniquely determined.

Denote by $x_{0}, x_{1}, \ldots, x_{n-1}$ the points of $P$ in their counterclockwise cyclic order on the boundary of the convex hull of $P$. In the following analysis the summation of indices is done modulo $n$. We claim that one can rename the points in $R$ to be $r_{0}, \ldots, r_{n-1}$ in such a way that for every $0 \leq i \neq j<n$ and $0 \leq k<n$ the point $x_{i}, x_{j}$, and $r_{k}$ are collinear if and only if $i+j+k=0$ (modulo $n$ of course). 


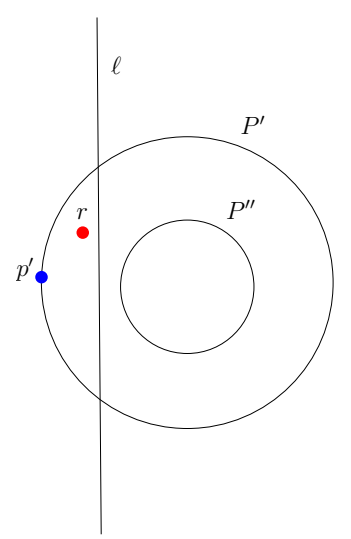

Figure 2: Illustration for the proof that no point of $R$ lies inside the convex hull of $P^{\prime}$ and outside the convex hull of $P^{\prime \prime}$.

Indeed, assume not, then one can find two distinct indices $i$ and $j$ such that $x_{i}$ and $x_{j}$ are collinear with $r_{k}$ for some $k$ while $x_{i+1}$ and $x_{j-1}$ are not collinear with $r_{k}$ and $i+1 \neq j-1$. Assume without loss of generality that $x_{i}$ lies between $r_{k}$ and $x_{j}$ (the case where $x_{j}$ lies between $r_{k}$ and $x_{i}$ is similar). Rotate the line through $r_{k}, x_{i}$, and $x_{j}$ about $r_{k}$ in the clockwise direction until the first time it meets a point in $P$. This point will be either $x_{i+1}$ or $x_{j-1}$ but not both at once. Then we find a line $\ell$ through $r_{k}$ and only one point of $P$ that does not support the convex hull of $P$. This is impossible because as we have seen every point $p \in P$ has a point in $r \in R$ that is not relevant for $p$ such that the line through $r$ and $p$ supports the convex hull of $P$ and this point $r$ is the unique point in $R$ that is not relevant for $p$. This completes the first step of the proof of Theorem 1.3 .

\subsection{Fitting $P \cup R$ into a cubic curve}

In the second step of the proof of Theorem 1.3 we will show that $P \cup R$ is contained in a cubic curve. We follow the approach of Green and Tao in GT13. The simple argument showing that $P \cup R$ is contained in a cubic curve relies on Chasles theorem:

Theorem 2.1 (Chasles Theorem). Let $\ell_{1}, \ell_{2}, \ell_{3}$ be three distinct lines that meet another family of three distinct lines $m_{1}, m_{2}, m_{3}$ at nine distinct intersection points. Then every cubic curve passing through 8 of these intersection points must pass also through the 9 'th.

As a direct consequence of Theorem 2.1 we get the following.

Claim 2.2. For $i \geq 0$, Any cubic passing through eight of the points $x_{n-1-i}, x_{n-2-i}, \ldots, x_{n-6-i}$ and $r_{5+2 i}, r_{7+2 i}, r_{9+2 i}$ must pass through all nine of them.

Proof. This is a direct application of Theorem 2.1. Take $\ell_{1}$ to be the line through $x_{n-2-i}, x_{n-3-i}$, and $r_{5+2 i}$. Take $\ell_{2}$ to be the line through $x_{n-4-i}, x_{n-5-i}$, and $r_{9+2 i}$. Take $\ell_{3}$ to be the line through $x_{n-1-i}, x_{n-6-i}$, and $r_{7+2 i}$. Take $m_{1}$ to be the line through $x_{n-3-i}, x_{n-6-i}$, and $r_{9+2 i}$. Take $m_{2}$ to be the line through $x_{n-1-i}, x_{n-4-i}$, and $r_{5+2 i}$. Take $m_{3}$ to be the line through $x_{n-2-i}, x_{n-5-i}$, and $r_{7+2 i}$. (See Figure 3)

Notice that the nine intersection points of a line from $\ell_{1}, \ell_{2}, \ell_{3}$ and a line from $m_{1}, m_{2}, m_{3}$ are $x_{n-1-i}, x_{n-2-i}, \ldots, x_{n-6-i}$, and $r_{5+2 i}, r_{7+2 i}$, and $r_{9+2 i}$. The assertion of the claim follows now from Theorem 2.1.

We use the easy and well known fact that for every nine points in the plane there is a cubic curve passing through all of them. Let $\Gamma$ be a cubic curve passing through $x_{n-1}, x_{n-2}, \ldots, x_{n-7}$ and also through $r_{5}$ and $r_{7}$. A repeated application of Claim 2.2 shows that $\Gamma$ must pass through 


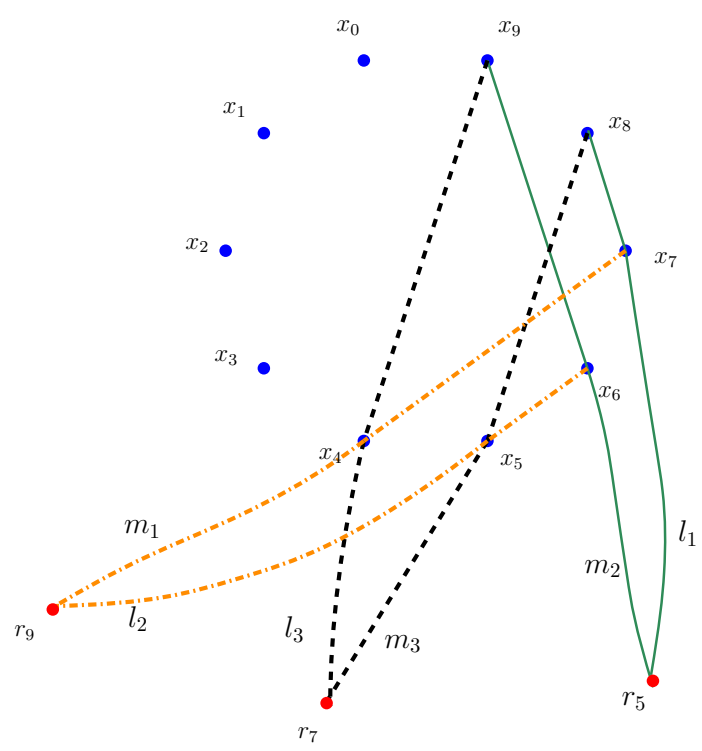

Figure 3: Illustration for the proof of Claim 2.2 with $n=10$ and $i=0$.

all the points $x_{0}, \ldots, x_{n-1}$ as well as through any point of the form $r_{5+2 i}$. If $n$ is odd, then this implies that all the points of $P \cup R$ are on $\Gamma$ and we are done. If $n$ is even this only implies that all the points of $P$ and the points $r_{1}, r_{3}, \ldots, r_{n-1}$ are on $\Gamma$ and we need to show that also $r_{0}, r_{2}, \ldots, r_{n-2}$ lie on $\Gamma$. This will follow from the following claim.

Claim 2.3. Any cubic passing through eight of the points $x_{n-1-i}, x_{n-2-i}, x_{n-3-i}, x_{n-5-i}, x_{n-6-i}, x_{n-7-i}$ and $r_{7+2 i}, r_{8+2 i}, r_{9+2 i}$ must pass through all nine of them.

Proof. This is a direct application of Theorem 2.1. Take $\ell_{1}$ to be the the line through $x_{n-1-i}, x_{n-6-i}$, and $r_{7+2 i}$. Take $\ell_{2}$ to be the line through $x_{n-2-i}, x_{n-7-i}$, and $r_{9+2 i}$. Take $\ell_{3}$ to be the line through $x_{n-3-i}, x_{n-5-i}$, and $r_{8+2 i}$. Take $m_{1}$ to be the line through $x_{n-3-i}, x_{n-6-i}$, and $r_{9+2 i}$. Take $m_{2}$ to be the line through $x_{n-2-i}, x_{n-5-i}$, and $r_{7+2 i}$. Take $m_{3}$ to be the line through $x_{n-1-i}, x_{n-7-i}$, and $r_{8+2 i}$. (See Figure 4)

Notice that the nine intersection points of a line from $\ell_{1}, \ell_{2}, \ell_{3}$ and a line from $m_{1}, m_{2}, m_{3}$ are $x_{n-1-i}, x_{n-2-i}, x_{n-3-i}, x_{n-5-i}, x_{n-6-i}, x_{n-7-i}$, and $r_{7+2 i}, r_{8+2 i}, r_{9+2 i}$. The assertion of the claim follows now from Theorem 2.1.

\section{A bipartite version of Theorem 1.3}

In this section we consider a bipartite version of Theorem 1.3 in which $P$ is a set of $2 n$ points that is the union of a set $B$ of $n$ blue and a set $G$ of $n$ green points. The set $R$ is a set of $n$ red points where we assume that the sets $B, G$, and $R$ are pairwise disjoint. We also assume that the set $P=B \cup G$ is in general position in the sense that no three of its points are collinear. Assume that every line through a point in $B$ and a point in $G$ contains also a point from $R$. We would like to show that $P \cup R$ lies on a cubic curve. We are able to show this under the additional assumption that whenever $b \in B, g \in G$, and $r \in R$ are collinear, then $r$ does not lie between $b$ and $g$.

Theorem 3.1. Let $B, G$, and $R$ be three pairwise disjoint sets of points in the plane. Assume that $B \cup G$ is in general position and $|B|=|G|=|R|=n$. If every line through a point $b \in B$ and a point $g \in G$ contains a point $r \in R$ that does not lie between $b$ and $g$, then $B \cup G \cup R$ lies on some cubic curve. 


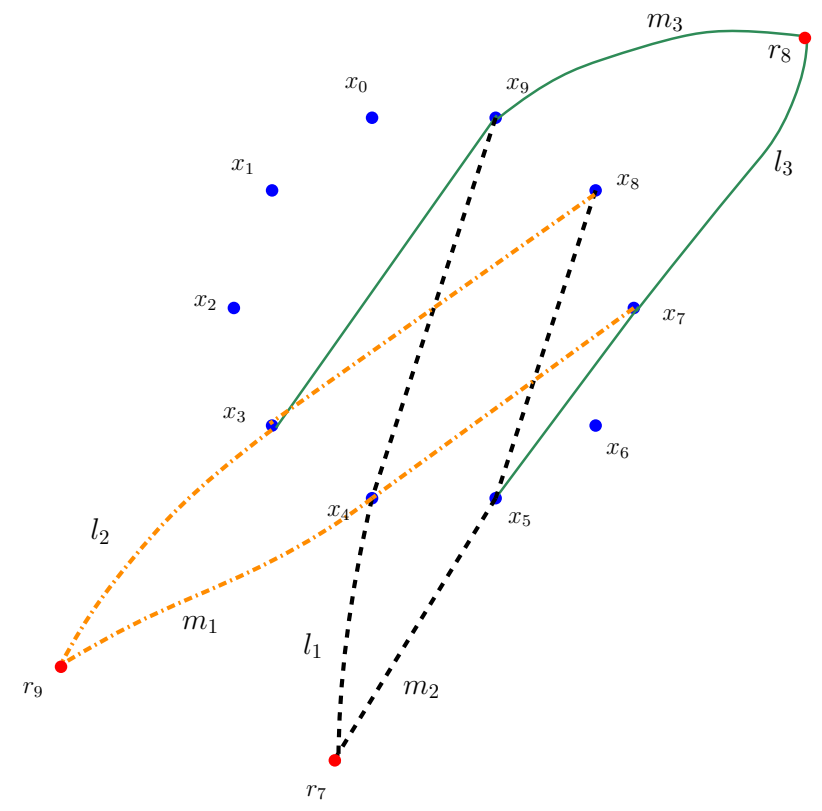

Figure 4: Illustration for the proof of Claim 2.3 with $n=10$ and $i=0$.

Proof. Here too the proof will consist of two steps. We first show that $B \cup G$ must be in convex position in such a way that the points of $B$ and $G$ are arranged alternately on the boundary of the convex hull of $B \cup G$. We will also be able to uniquely determine the structure of the collinear triples of a point from $B$, a point from $G$, and a point from $R$. In the second step we will use Chasles theorem to conclude that $B \cup G \cup R$ lie on a cubic curve.

One easy but crucial observation is that any line through two points of different colors in $B \cup G \cup R$ must pass through through a unique point of the third color. This is because $B \cup G$ is in general position, $|B|=|G|=|R|=n$, and there is a point in $R$ on each one of the $n^{2}$ lines through a blue point and a green point.

We split into two possible cases:

Case 1. On the boundary of the convex hull of $B \cup G$ there are only points of the same color, without loss of generality let it be blue. We will show that this case is impossible. Let $\ell$ be a line supporting the convex hull of $B \cup G$ in an edge whose vertices are $b_{1}, b_{2} \in B$. Rotate $\ell$ about $b_{1}$ so that it separates $b_{2}$ from the rest of the points in $B \cup G$ until the first time it meets a point $g$ in $G$. There is a point $r \in R$ on the line through $b_{1}$ and $g$, and $g$ must lie between $b_{1}$ and $r$ on that line. To obtain a contradiction observe that the line through $r$ and $b_{2}$ cannot contain a point $g^{\prime}$ of $G$ in such a way that $r$ does not lie between $b_{2}$ and $g^{\prime}$ on that line.

Case 2. On the boundary of the convex hull of $B \cup G$ there are points of both colors blue and green. We claim that along the boundary of the convex hull of $B \cup G$ blue and green points appear alternately. To see this, notice that the contrary assumption is, without loss of generality, that one can find three consecutive vertices along the convex hull of $B \cup G$ such that the first one $b_{1}$ is blue while the next two $g_{1}$ and $g_{2}$ are green. Consider the line $\ell$ through $b_{1}$ and $g_{2}$. It must contain a point $r \in R$ that lies outside the convex hull of $B \cup G$. We get a contradiction as the line through $r$ and $g_{1}$ cannot contain any point of $B$.

Next we show that the points of $B \cup G$ must be in convex position. Denote by $k$ the number of blue points that is also the same as the number of green points on the boundary of the convex hull of $B \cup G$. Notice that every point of $R$ outside the convex hull of $B \cup G$ must be collinear with unique two edges of the convex hull of $B \cup G$. Therefore, there are precisely $k$ points of $R$ outside the convex hull of $B \cup G$. Moreover, these $k$ points of $R$ are exactly those points of $R$ 


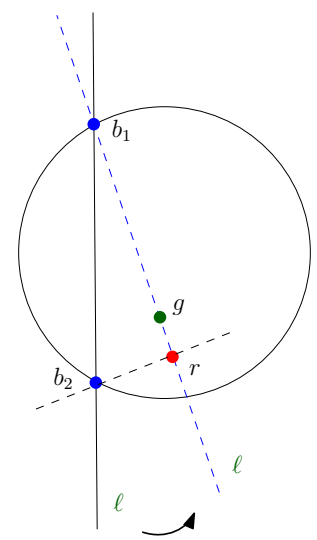

Figure 5: Illustration for the proof of Theorem 3.1 .

that pierce all the $k^{2}$ lines through two points of different colors on the boundary of the convex hull of $B \cup G$. Therefore, if $r \in R$ lies outside the convex hull of $B \cup G$, then any line passing through $r$ and a point on the boundary of the convex hull of $B \cup G$ must pass through another point of different color also lying on the boundary of the convex hull of $B \cup G$.

In order to show that the points of $B \cup G$ are in convex position it is enough to show that there is no point of $G$ inside the convex hull of $B \cup G$. Let $b_{1}$ and $b_{2}$ be two blue points on the boundary of the convex hull of $B \cup G$. Let $\ell$ be the line through $b_{1}$ an $b_{2}$. (See Figure 5) It is enough to show that as we rotate $\ell$ in the counterclockwise direction around $b_{1}$, as long as $\ell$ does not contain an edge of the convex hull of $B \cup G$, it never meets a point of $G$ inside the convex hull of $B \cup G$ (then we can change the role of $b_{1}$ and $b_{2}$ ). Assume to the contrary that $g \in G$ is the first such point let $r$ be the point of $R$ on the line through $b_{1}$ and $g$. The point $r$ cannot lie outside of the convex hull of $B \cup G$ because the line through $r$ and $b_{1}$ does not contain a green point on the boundary of the convex hull of $B \cup G$. Now consider the line through $r$ and $b_{2}$. We get a contradiction as this line cannot contain a green point $g^{\prime}$ in such a way that $r$ does not lie between $b_{2}$ and $g^{\prime}$ on that line.

Having shown that the points of $B \cup G$ lie in convex position and alternately, let us denote the points of $B \cup G$ by $x_{0}, x_{1}, \ldots, x_{2 n-1}$ according to their cyclic order on the boundary of the convex hull of $B \cup G$. We claim that for every odd number $k$ between 0 and $2 n$ there is a unique point $r$ (that we will denote by $r_{2 n-k}$ ) such that $r$ is collinear with all the pairs $x_{i}$ and $x_{j}$ such that $i+j=k$ (modulo $2 n$ ). Indeed, under the contrary assumption one can find $i$ and $j$ such that $i+j=k, x_{i}$ and $x_{j}$ are collinear with $r \in R$ but at the same time $r$ is not collinear with $x_{i+1}$ and $x_{j-1}$. To get a contradiction rotate the line $\ell$ through $x_{i}$ and $x_{j}$ about $r$ such that it separates $x_{i}$ and $x_{j}$ from $x_{i+1}$ and $x_{j-1}$ until the first time it hits a point in $B \cup G$. This point must be either $x_{i+1}$ or $x_{j-1}$ and no other point of $B \cup G$ lies on that line, a contradiction.

Therefore, after denoting the points of $R$ by $r_{1}, r_{3}, \ldots, r_{2 n-1}$, as suggested above, we see that $x_{i}, x_{j}$, and $r_{k}$ are collinear iff $i+j+k=0$ modulo $2 n$.

Now comes the second part of the proof where we will use Chasles theorem (in the form of Claim 2.2 to show that $B \cup G \cup R$ lie on a cubic.

Let $\Gamma$ be a cubic curve passing through $x_{2 n-1}, x_{2 n-2}, \ldots, x_{2 n-7}$ as well as through $r_{5}$ and $r_{7}$. A repeated application of Claim 2.2 (with $2 n$ in the role of $n$ in the statement of Claim 2.2) shows that all the points $x_{0}, x_{1}, \ldots, x_{2 n-1}$ and $r_{1}, r_{3}, r_{5}, \ldots, r_{2 n-1}$ must lie on $\Gamma$.

\section{References}

$\left[\mathrm{ABK}^{+} 08\right]$ E. Ackerman, K. Buchin, C. Knauer, R. Pinchasi, and G. Rote. There are not too many magic configurations. Discrete \& Computational Geometry, 39(1):3-16, 2008. 
[BMM14] A. Blokhuis, G. Marino, and F. Mazzocca. Generalized hyperfocused arcs in PG(2,p). Journal of Combinatorial Designs, 22(12):506-513, 2014.

[BMP05] P. Brass, W. O. J. Moser, and J. Pach. Research Problems in Discrete Geometry. Springer US, 2005.

[Cha70] G. D. Chakerian. Sylvester's problem on collinear points and a relative. Amer. Math. Monthly, 77:164-167, 1970.

[Ele99] G. Elekes. On linear combinatorics III. Few directions and distorted lattices. Combinatorica, 19(1):43-53, 1999.

[EP78] P. Erdős and G. Purdy. Some combinatorial problems in the plane. Journal of Combinatorial Theory, Series A, 25(2):205-210, 1978.

[Grü75] B. Grünbaum. Arrangements of colored lines, abstract 720-50-5. Notices Amer. Math. Soc., 22:A-200, 1975.

[GT13] B. Green and T. Tao. On sets defining few ordinary lines. Discrete $\mathscr{G}$ Computational Geometry, 50(2):409-468, 2013.

[Jam85] R. E. Jamison. A survey of the slope problem. Ann. New York Acad. Sci., 440(1):34$51,1985$.

[Jam86] R. E. Jamison. Few slopes without collinearity. Discrete Mathematics, 60:199-206, 1986.

[Mil18] L. Milićević. Classification theorem for strong triangle blocking arrangements. arXiv:1809.08639, 2018.

[Mot67] T. S. Motzkin. Nonmixed connecting lines, abstract 67T 605. Notices Amer. Math. Soc., 14:837, 1967.

[Mur71] U. S. R. Murty. How many magic configurations are there? Amer. Math. Monthly, 78(9):1000-1002, 1971.

[Pil18] C. Pilatte. On the sets of $n$ points forming $n+1$ directions. arXiv:1811.01055, 2018.

[Pin13] R. Pinchasi. A solution to a problem of Grünbaum and Motzkin and of Erdős and Purdy about bichromatic configurations of points in the plane. Israel Journal of Mathematics, 198(1):205-214, 2013.

[Pin18] R. Pinchasi. Stabbing all lines determined by points in general position in the plane. Manuscript, 2018.

[PP19] R. Pinchasi and A. Polyanskii. A one-page solution of a problem of Erdős and Purdy. Manuscript, 2019.

[Sco70] P. R. Scott. On the sets of directions determined by $n$ points. Amer. Math. Monthly, 77(5):502-505, 1970.

[Ung82] P. Ungar. $2 n$ noncollinear points determine at least $2 n$ directions. J. Combin. Th. Ser. A, 33(3):343-347, 1982. 